\title{
Intercalation of Graphene on Iridium with Sodium Atoms
}

\author{
E. V. Rut'kov ${ }^{a}$ and N. R. Gall' $a, b, c, *$ \\ ${ }^{a}$ Ioffe Physical-Technical Institute, Russian Academy of Sciences, \\ Politekhnicheskaya ul. 26, St. Petersburg, 194021 Russia \\ ${ }^{b}$ Institute for Analytical Instrumentation, Russian Academy of Sciences, \\ ul. Ivana Chernykh 31-33, lit. A, St. Petersburg, 198095 Russia \\ ${ }^{c}$ St. Petersburg Polytechnic University, Politekhnicheskaya ul. 29, St. Petersburg, 195251 Russia \\ *e-mail:gall@ms.ioffe.rssi.ru \\ Received November 17, 2014; in final form, December 13, 2014
}

\begin{abstract}
It has been shown that sodium atoms deposited on the surface of graphene atop iridium at $T \leq$ $850 \mathrm{~K}$ diffuse under the graphene layer into an intercalated state and accumulate there in significant concentrations $\sim(2-3) \times 10^{14}$ atoms cm ${ }^{-2}$. The release of the atoms from under the graphene "carpet" takes place upon destruction of the layer at $T \geq 1800 \mathrm{~K}$. The physical nature of the differences in the processes of release of atoms of different alkali metals from under graphene has been discussed.
\end{abstract}

DOI: $10.1134 / \mathrm{S} 1063783415060281$

\section{INTRODUCTION}

The effect of intercalating graphene films atop a metal (iridium) surface with potassium atoms, which consists in the penetration (diffusion) of these atoms into the space between the metal surface and graphene film, was revealed for the first time in our earlier work [1]. Then, the regularities of intercalation of graphene films deposited on various substrates ( $\mathrm{Pt}, \mathrm{Rh}, \mathrm{Re}, \mathrm{Mo})$ were studied for many atoms ( $\mathrm{Cs}, \mathrm{Si}, \mathrm{Ir}, \mathrm{C}, \mathrm{Al}, \mathrm{Ni}, \mathrm{Ba}$, $\mathrm{Pt}, \mathrm{Ag}, \mathrm{Cu}$ ) and also for molecules of $\mathrm{C}_{60}$ fullerene [2, $3]$. In the recent years, this problem has been studied by many research groups which considered the intercalation of graphene layer atop $\operatorname{Ir}(111)$ with atoms $\mathrm{Si}$ [4], $\mathrm{Cu}$ [5], $\mathrm{Hf}$ [6], and also $\mathrm{Li}$ atoms for graphene on a silicon carbide substrate [7]. As for bulk compounds, this effect has usually been observed in layered solids (graphite, $\mathrm{MoS}_{2}$ ) when some atoms and molecules are embedded into interlayer space [8-11]. For example, during exposure of a heated graphite crystal to cesium vapors, cesium spontaneously penetrates between graphite layers, forming intercalated compound $\mathrm{C}_{8} \mathrm{Cs}$, in which graphite layers with concentration $N_{\mathrm{C}}=3.86$ $\times 10^{15} \mathrm{~cm}^{-2}$ alternate with cesium layers with $N_{\mathrm{Cs}}=5 \times$ $10^{14} \mathrm{~cm}^{-2}$, and the distance between the neighboring graphite layers increases from $3.35 \AA$ in the graphite single crystal to $5.94 \AA$ in $\mathrm{C}_{8} \mathrm{Cs}$ [8]. It is interesting and important to note that the intercalation does not change the structure and lattice parameter of the graphite layer and, thus, its individuality. It is interesting that it is $\mathrm{Na}$ that forms the least stable intercalated compounds among all alkali metals $[9,10]$.

In [12-17], the regularities of intercalating singlelayer graphene layers atop $\operatorname{Ir}(111)$ and $\operatorname{Re}(10-10)$ with atoms of some alkali metals (Cs, K), multivalent atoms with high ionization polarizations $(\mathrm{Pt}, \mathrm{Si}, \mathrm{C}, \mathrm{Ag}, \mathrm{Al}$, Ir, $\mathrm{Mo}, \mathrm{Cu}, \mathrm{Ba}$ ), as well as with molecules of $\mathrm{C}_{60}$ fullerene, were studied in details. It was shown in these works that it is the ionization potential of an intercalating atom $e V$ that is the factor separating two qualitatively different variants of intercalation: "single-layer" intercalation inherent to atoms with low ionization polarizations $e V$ and "multilayer" intercalation that is characteristic of multivalent atoms. Similar multilayer intercalation is observed during adsorption of gold on graphene layer atop $\mathrm{Ni}(111)$ [18] and during intercalation of graphene layers into graphite [19]. The aim of this work was to investigate the intercalation of graphene with sodium atoms that have the highest ionization potential $e V_{\mathrm{Na}}=5.14 \mathrm{eV}$, as compared to cesium and potassium $e V_{\mathrm{Cs}}=3.89 \mathrm{eV}$ and $e V_{\mathrm{K}}=$ $4.34 \mathrm{eV}$, but substantially smaller atomic and ionic radii [20].

\section{SAMPLE PREPARATION AND EXPERIMENTAL TECHNIQUE}

The experiments were performed in two ultrahigh-vacuum setups: in a high-resolution Auger spectrometer $(\Delta E / E \sim 0.1 \%)$ with a prism energy analyzer, in which surface layers were studied by the electron Auger spectroscopy, surface ionization and thermionic emission (TESI method) [21], and in a magnetic mass-spectrometer, in which the TESI method was used with an additional possibility of the detection of desorbing neutral atoms due to their ionization by electron impact [22]. We used directly heated $50 \times 1 \times$ $0.02-\mathrm{mm}$ iridium ribbons which were textured by an alternating current. The (111) face with work function $e \varphi=5.75 \mathrm{eV}$ emerged on the surface, and the surface 


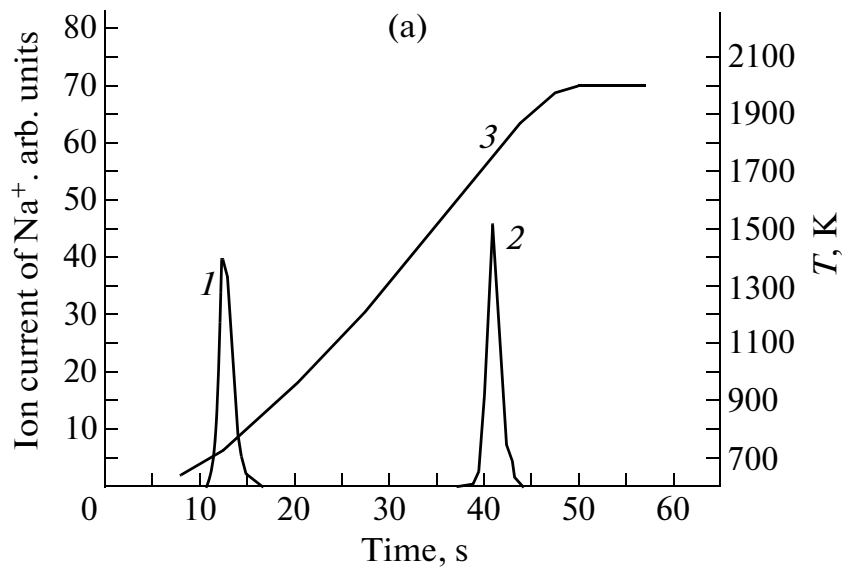

(b)

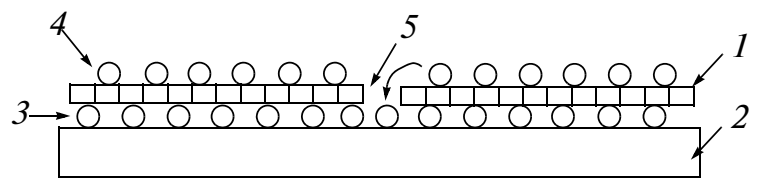

Fig. 1. Release of sodium atoms from the intercalated state under graphene atop iridium. (a) Thermal desorption spectra of sodium atoms during heating the ribbon from room temperature: $(1,2)$ desorption phases and (3) time dependence of the temperature $T(t)$. The coating was obtained by deposition of sodium at $T=300 \mathrm{~K}$ on graphene atop iridium by the flux of sodium atoms $v_{\mathrm{Na}}=$ $3 \times 10^{12} \mathrm{~cm}^{-2} \mathrm{~s}^{-1}$ for $60 \mathrm{~s}$; (b) model representation of (1) graphene layer on (2) iridium with (3) intercalated and (4) adsorbed sodium atoms; (5) graphene layer defect.

was uniform in $e \varphi$. According to X-ray diffraction data, the degree of face orientation was $99.8 \%$. According to scanning electron microscopy, the average grain size in the surface plane was $\sim 50 \mu \mathrm{m}$.

To prepare graphene film on iridium, we introduced benzene vapors into the equipment from a special leak-in system. Benzene dissociates on the heated ( $T=1700-1900 \mathrm{~K})$ iridium, hydrogen is desorbed, and carbon is retained on the surface and form graphene islands on it; the area of the islands increases, they coalesce and form a two-dimensional carbon layer of graphite structure, namely, graphene. The process of formation of graphene was studied in detail in $[17,18,21]$. Scanning tunneling microscopy confirmed the formation of a continuous graphene film $[23,24]$. Note that the graphene film atop $\operatorname{Ir}(111)$ is actively disrupted at temperatures $1800-1900 \mathrm{~K}$; at $T>2000 \mathrm{~K}$, carbon is desorbed from the surface [25].

The sodium atom flux was fed onto the surface from a Knudsen cell; the flux density was determined in special experiments using the method of surface ionization in the case when $e \varphi \gg e V_{\mathrm{Na}}$ : for example, in the case of ionization of sodium atoms on a clean iridium surface, their $100 \%$ ionization takes place [26]. In the experiments, sodium ions were completely accumulated from the central part of the ribbon, and the

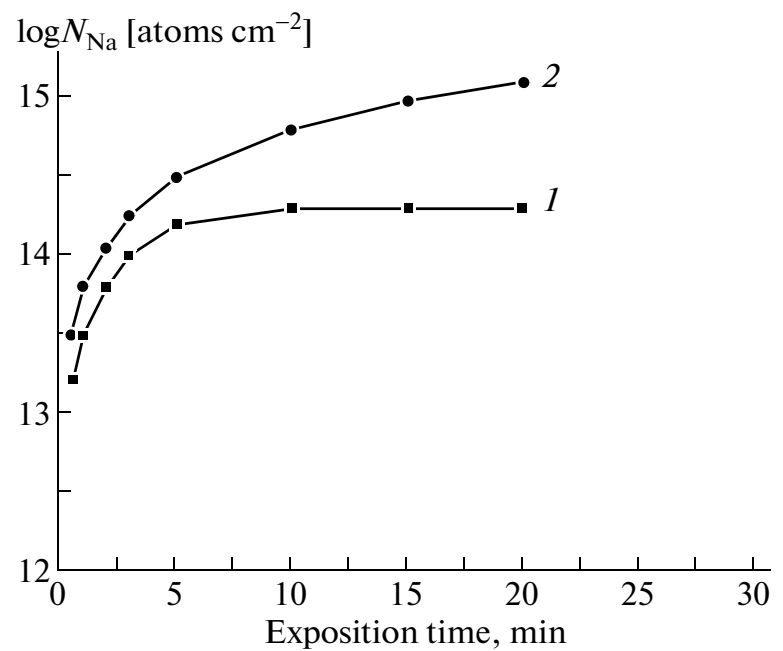

Fig. 2. (1) Dependence of the logarithm of the concentration of sodium atoms $\log N_{\mathrm{Na}}$ in the phase on the time of exposure of the iridiumgraphene system at $T=300 \mathrm{~K}$ in the flux of sodium atoms $v_{\mathrm{Na}}=1 \times 10^{12}$ atoms cm $\mathrm{cm}^{-2} \mathrm{~s}^{-1}$ and (2) dependence of the deposited sodium dose $\log N_{\mathrm{Na}}$, where $N_{\mathrm{Na}}=v_{\mathrm{Na}} t$ on the exposure time.

measured current of these ions $I_{\mathrm{Na}}^{+}$was determined using relationship

$$
I_{\mathrm{Na}}^{+}=e \mathrm{v}_{\mathrm{Na}} S .
$$

This allowed us to determine the flux density of the deposited sodium: $v_{\mathrm{Na}}=I_{\mathrm{Na}}^{+} / e S$, where $e$ is the electron charge and $S$ is the area of accumulating sodium ions; in our case, $S=5 \mathrm{~mm}^{2}$.

\section{RESULTS AND DISCUSSION}

Sodium atoms were adsorbed on graphene layer atop iridium ( $\mathrm{Ir}-\mathrm{C}$ system) at $300 \mathrm{~K}$, and then the system was flash-heated. The thermodesorption spectrum contained two desorption phases (Fig. 1a). The first $\alpha$ phase ( 1 in Fig. 1a) is due to desorption of $\mathrm{Na}$ atoms from graphene surface (4 in Fig. 1b) at $T \sim$ $800 \mathrm{~K}$ : the desorption temperature agrees well with the lifetime of $\mathrm{Na}$ adatoms on graphene [27]. The second high-temperature $\gamma$ phase is due to escaping sodium atoms from under graphene layer (3 in Fig. 1b) and corresponds to a temperature $T \geq 1750 \mathrm{~K}$ ( 2 in Fig. 1a). Qualitatively similar desorption phases were observed during intercalating graphene-coated $\operatorname{Ir}(111), \operatorname{Re}(10-$ 10 ), and $\mathrm{Rh}(111)$ with Cs and $\mathrm{K}$ atoms [13]; however, the region of escaping the particles from under the layer ( $\gamma$ phase) for these particles shifted to higher temperatures as compared to that of sodium.

Figure 2 shows the kinetics of filling of the $\gamma$ phase with sodium at $T=300 \mathrm{~K}$. It is seen that, at $t=6 \mathrm{~min}$, the sodium concentration in the $\gamma$ phase reaches the saturation corresponding to $N_{\mathrm{Na}} \sim(2-3) \times 10^{14} \mathrm{~cm}^{-2}$ 
( 1 in Fig. 1a). Curve 2 shows the dependence of the total number of sodium atoms falling on the surface, i.e., the deposited dose $N_{\mathrm{Na}}=v_{\mathrm{Na}} t$. Thus, sodium, as well as other alkali metals, is accumulated under graphene with the concentration not higher than one monoatomic layer, and one more layer is adsorbed on its external surface. Recall that, conversely, atoms with high ionization potentials such as $\mathrm{Pt}$ atoms intercalate under graphene coating $\operatorname{Ir}(111)$ in unlimited amounts at $T=1100-1200 \mathrm{~K}$, and, in this case, graphene remains adsorbate-free [13].

Figure 3 shows the dependence of the number of sodium atoms under graphene layer which are accumulated for the same time $t=3 \mathrm{~min}$ and a constant deposition flux but at various substrate temperatures. Each point in the curve was obtained as a result of new experiment: "old" sodium was removed by a flash, graphene layer was grown anew, and the measurements were performed at a new temperature. It is seen that a marked number of sodium atoms fall under graphene "carpet" only at $T \leq 850 \mathrm{~K}$, as well as in the case of other studied alkali metals (Cs and K) [2, 13]. Likely, this effect has a general character and is due to electrostatic repulsion of charged adatoms of alkali metals, which favors their leaving through graphene layer defects for the intercalated state [13]. For the electrostatics to start to play a part, the adsorbate concentration on the graphene surface must be marked, which needs a decrease in temperature: at $T>900 \mathrm{~K}$, the equilibrium concentration of alkali metal atoms on the graphene surface is very low [27].

A small number of $\mathrm{Na}$ atoms accumulated under graphene at $T>900 \mathrm{~K}(\sim 0.01 \%$ of the total number of atoms falling on the surface) can be explained assuming that they are atoms which directly fall on graphene layer defects with subsequent diffusion under the layer ( 5 in Fig. 1b). It should be noted as well that during falling the adsorbate on the surface, the adsorption capacities increase for both the phases observed in the thermal desorption: the phase related to escaping from under the layer and the phase forming as a result of the adsorbate desorption from the graphene external surface. This fact has a total character for all alkali metals.

Atoms of all alkali metals in intercalated state influence the work function of graphene decreasing it by several tenth electron-volts. However, in the case of the same adsorbate concentration under graphene $\sim 2 \times 10^{14} \mathrm{~cm}^{-2}, \Delta e \varphi \sim 0.3 \mathrm{eV}$ for Cs and $\Delta e \varphi \sim 0.08 \mathrm{eV}$ for $\mathrm{Na}$. The same concentration of the adsorbate on the graphene surface decreases the work function by $\sim 1.5-2 \mathrm{eV}$. The model explaining the causes of easy entering of alkali metal adatoms under graphene $(T<$ $800 \mathrm{~K})$ and hampered escaping them from under graphene ( $T \gg 800 \mathrm{~K})$ was considered in [13], and it is related to the electrostatic repulsion of positively charged adatoms on and under graphene.

Let us summarize the laws of intercalation of graphene-coated metals with alkali atoms of $\mathrm{Na}, \mathrm{K}$,

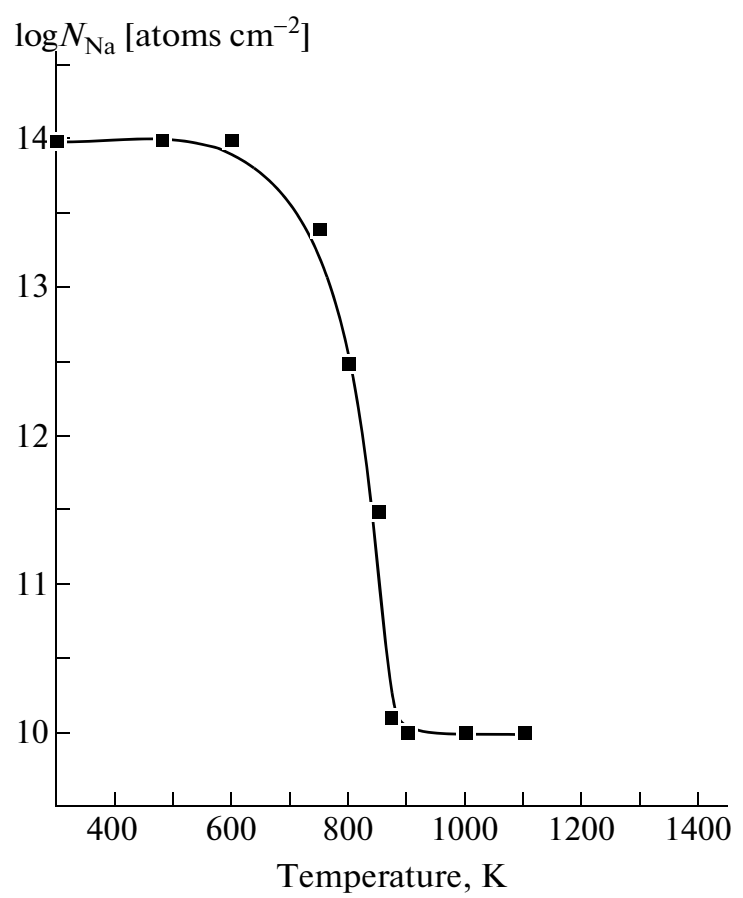

Fig. 3. Dependence of the concentration of sodium atoms $\log N_{\mathrm{Na}}$ under the graphene layer atop iridium on the substrate temperature during the exposure of the sample in the flux of sodium atoms $v_{\mathrm{Na}}=7 \times 10^{11}$ atoms $\mathrm{cm}^{-2} \mathrm{~s}^{-1}$ for $3 \mathrm{~min}$. Each point was obtained in an individual experiment.

Cs, indicating general features and observed differences:

(1) At $N \geq 10^{13} \mathrm{~cm}^{-2}$, the $\gamma$ phase begins to be filled; i.e., adatoms diffuse under the graphene "carpet", and the process occurs at room temperature. It is evident that the increase in the concentration of the ionized adsorbate existing on the surface causes the activation of the surface migration of adatoms stimulated by the Coulomb repulsion that provides the experimentally observed migration release of adatoms to the back side of the ribbon. Therefore, as temperature increases, the efficiency of intercalation decreases, and the intercalation is not almost observed at temperatures $T>700-$ $800 \mathrm{~K}$, at which the coating with alkali-metal atoms becomes negligible small.

(2) The graphene "carpet" confines intercalated atoms, and this fact shifts the temperature threshold of removing the adsorbate by more than $1000 \mathrm{~K}$ as compared to that of pure metallic surface. In this case, the temperature of escaping the intercalate from under graphene coating iridium surface increases in the series $\mathrm{Na} \longrightarrow \mathrm{K} \longrightarrow$ Cs.

(3) Alkali metals decrease the work function of graphene, and their effect increases in the series $\mathrm{Na} \longrightarrow \mathrm{K} \longrightarrow$ Cs.

Note as well that, in the case of intercalated cesium, we observed the effect of increase in the ther- 
mal stability of graphene layer, consisting in the shift of the destruction temperature of graphene by approximately 100-200 K toward higher temperatures. The mechanism of this phenomenon is considered in [17]. No this effect is observed for sodium and potassium.

\section{CONCLUSIONS}

The intercalation mechanism, i.e., penetration of atoms under the graphene film, is the same for $\mathrm{Na}, \mathrm{K}$, $\mathrm{Cs}$ and is based on the electrostatic repulsion of individual adsorbate atoms on the external graphene surface, which agrees well with the existing concepts of the intercalation of bulk graphite with alkali metal atoms [10]. Conversely, the release of atoms from the intercalated state corresponds to the specificity of their interaction not only with graphene but also with the metal surface.

The observed differences, such as, first, the earlier release of sodium from under graphene during heating, are likely due to a smaller sodium atom radii, which allow them to freely migrate under graphene, and, second, are likely due to smaller effective charge of these atoms in the intercalated state, which decreases the role of collective processes which hamper the migration. The above-mentioned causes are also possible to lead to a weaker influence of sodium as compared to that of cesium on the work function of the graphene layer under which these atoms are present.

\section{REFERENCES}

1. E. V. Rut'kov and A. Ya. Tontegode, Sov. Tech. Phys. Lett. 7 (10), 480 (1981).

2. E. V. Rut'kov and A. Ya. Tontegode, Phys.-Usp. 36 (11), 1053 (1993).

3. N. R. Gall, E. V. Rut'kov, and A. Ya. Tontegode, Mod. Phys. B 11, 1865 (1997).

4. I. Meng, R. Wa, H. Zhou, G. Lis, Yi Zhang, L. Li, Y. Wang, and H. J. Gao, Appl. Phys. Lett. 100, 083101 (2012).

5. M. Sicot, Y. Fagot-Revurat, B. Kierren, G. Vasseur, and D. Malterre, Appl. Phys. Lett. 105, 191603 (2014).

6. L. Li, Y. Wang, I. Meng, R. Wa, and H. J. Gao, Appl. Phys. Lett. 102, 093196 (2013).

7. C. Virojanadara, S. Watcharinyanon, A. Zakharov, and L. Johansson, Phys. Rev B: Condens. Matter 82, 205402 (2010).
8. H. Zabel and S. A. Solin, Graphite Intercalation Compounds, Vol. 1: Structure and Dynamics (Springer-Verlag, Berlin, 1990).

9. J. E. Fisher, Physica B+C (Amsterdam) 99, 383 (1980).

10. M. S. Dresselhaus and G. Dresselhaus, Adv. Phys. 30 139 (1981).

11. M. T. John and H. U. Stranberg, Solid State Commun. 57, 545 (1986).

12. N. A. Kholin, E. V. Rut'kov, and A. Ya. Tontegode, Surf. Sci. 139, 155 (1984).

13. N. R. Gall', E. V. Rut'kov, and A. Ya. Tontegode, Sov. Tech. Phys. Lett. 14 (3), 235 (1988).

14. N. R. Gall, S. N. Mikhailov, E. V. Rut'kov, and A. Ya. Tantegode, Synth. Met. 34, 447 (1989).

15. N. R. Gall, S. N. Mikhailov, E. V. Rut'kov, and A. Ya. Tontegode, Surf. Sci. 226, 381 (1990).

16. E. V. Rut'kov, A. Ya. Tontegode, M. M. Usufov, and Yu. S. Grushko, Mol. Mater. 4, 217 (1994).

17. N. R. Gall and E. V. Rut'kov, in Physics and Applications of Graphene: Experiments, Ed. by S. Mikhailov (InTech, Rijeca, Croatia, 2011), p. 293.

18. A. M. Shikin, Formation, Electronic Structure, and Properties of Low-Dimensional Structures Based on Metals (VVM, St. Petersburg, 2011) [in Russian].

19. M. Fauchard, S. Cohen, P. Lagrange, and J. F. Mareche, Carbon 65, 236 (2013).

20. Properties of Elements: A Reference Book, Ed. by M. E. Drits (Metallurgiya, Moscow, 1985) [in Russian].

21. A. Ya. Tontegode, Prog. Surf. Sci. 38, 201 (1991).

22. E. Ya. Zandberg, E. V. Rut'kov, and A. Ya. Tontegode, Sov. Phys. Tech. Phys. 21 (12), 1541 (1976).

23. Z. Waqar, I. V. Makarenko, A. N. Titkov, N. R. Gall, E. V. Rut'kov, and A. Ya. Tontegode, J. Mater. Res. 19, 1058 (2004).

24. Z. Kluzek, W. Kozlowski, Z. Waqar, S. Patta, J. S. Buenell-Gray, I. V. Makarenko, N. R. Gall, E. V. Rut'kov, A. Ya. Tontegode, and A. N. Titkov, Appl. Surf. Sci. 252, 1221 (2005).

25. N. R. Gall', M. S. Kobrin, S. N. Mikhailov, E. V. Rut'kov, and A. Ya. Tontegode, Poverkhnost 9, 21 (1984).

26. E. Ya. Zandberg and N. I. Ionov, Surface Ionization (Nauka, Moscow, 1969; Israel Program for Scientific Translations, Jerusalem, 1971).

27. N. R. Gall and E. V. Rut'kov, in Physics and Applications of Graphene: Experiments, Ed. by S. Mikhailov (InTech, Rijeca, Croatia, 2011), p. 209.

Translated by Yu. Ryzhkov 